\title{
Anisotropic size effect in strength in coherent nanowires with tilted twins
}

\author{
Yujie Wei* \\ State Key Laboratory of Nonlinear Mechanics, Institute of Mechanics, Chinese Academy of Sciences, Beijing 100190, P.R. China
}

(Received 24 May 2011; published 22 July 2011)

\begin{abstract}
When materials are deformed plastically via dislocations, a general finding is that samples with smaller dimensions exhibit higher strengths but with very limited amount of plasticity in tension. Here we report that one-dimensional coherent nanostructures with tilted internal twins exhibit anisotropic size effect: their strengths show no apparent change if only their thicknesses reduce, but become stronger as the sample sizes are reduced proportionally. Large-scale molecular dynamics simulations show that such nanowires deform primarily through twin migration mediated by partial dislocations in one active slip system, and a large amount of plasticity could be achieved in such nanowires via twin migration. The unique structure shown here is suitable to explore strengthening mechanisms in metals when plasticity is controlled by a single dislocation slip system. This study also suggests a novel approach to modulate strength and ductility in one-dimensional coherent nanostructures.
\end{abstract}

DOI: 10.1103/PhysRevB.84.014107

PACS number(s): 62.25.-g, 62.20.F-, 62.23.St

\section{INTRODUCTION}

A thorough understanding of deformation mechanisms in one-dimensional nanostructures is critical for further device miniaturization in all technological fields. Recent progress in fabrication and testing of micro- and nanoscale metallic or intermetallic pillars or wires have generally confirmed the notion of smaller being stronger-the strengths of materials tend to increase as either their sample sizes or microstructural dimensions decrease. The work of Uchic et al. ${ }^{1}$ showed that single-crystal nickel and intermetallic $\mathrm{Ni}_{3} \mathrm{Al}$-Ta samples with smaller radii exhibit higher yield strengths. Subsequently, similar size effects were revealed in other metallic systems ${ }^{2-11}$ and discussed in recent review articles. ${ }^{12,13}$ Here we report from large-scale molecular dynamics (MD) simulations that coherent $\mathrm{Cu}$ nanowires (NWs) with tilted internal twins (see Fig. 1 for illustration) show anisotropic size effect: their strengths are insensitive to the decrease in sample thicknesses in [110] direction, but exhibit smaller-being-stronger effect as all dimensions of the sample are proportionally reduced. Such NWs also show large amount of plasticity in tension, and plastic deformation is exclusively accommodated by twin migration, i.e. partial dislocation mediated detwinning. We note that the detwinning mechanism mediated large plasticity in NWs with tilted twins differs from pseudoelasticity induced by crystal reorientation facilitated by the formation of twin boundaries in NWs with diameters less than $5 \mathrm{~nm} .{ }^{14-18}$

Previous investigations on one-dimensional twinned NWs are for the case that twin planes are aligned to be perpendicular to the wire axes. ${ }^{19-21}$ When such NWs are subjected to axial loading, the resolved shear stresses within the twin planes remain zero. It effectively deactivated three important slip systems parallel to the twin planes; only dislocations inclined to twin planes are activated, and deformation by dislocation motion within the twin planes are prohibited. In contrast, NWs with tilted internal twins (see Fig. 1(a)) allow us to include dislocation activities in all slip systems. In fact, we will show that by choosing an appropriate twin orientation with respect to the wire axis, it is possible to design the behaviors of NWs such that its deformation is dominated by only one slip system parallel to the twin planes. To circumvent the complexity of highly disordered surface structures in cylindrical samples, we consider NWs with rectangular cross sections, where potential sites for dislocation nucleation reside at the junctions of twin planes with sample ledges. Characteristic dimensions of the sample include wire width $W$, wire thickness $h$, and twin spacing $\lambda$. We note that with current experimental techniques in nanoscale tensile testing, ${ }^{22-24}$ it should be feasible to fabricate NWs with tilted twins shown in Fig. 1(a).

\section{METHODS FOR MOLECULAR DYNAMICS SIMULATIONS}

The simulations are performed on both two-dimensional and three-dimensional NWs with pre-existing twins with tilted angle $\theta=30^{\circ}$. In each sample, the width-over-length ratio is kept to be 3:40 to ensure sufficient twins. The length of respective sample is hence $400 \mathrm{~nm}$ for $W=30 \mathrm{~nm}, 280 \mathrm{~nm}$ for $W=21 \mathrm{~nm}, 160 \mathrm{~nm}$ for $W=12 \mathrm{~nm}$, and $80 \mathrm{~nm}$ for $W=6 \mathrm{~nm}$. Twins and matrix are equally spaced, which leads to exactly $50 \%$ of twinned volume in a NW. The samples with length 400 $\mathrm{nm}$, width $W=30 \mathrm{~nm}$, and thickness $h=25.6 \mathrm{~nm}$ contains $25,971,600$ atoms. For each wire width, five initially uniform twin spacings $\lambda=0.626,1.88,6.26,11.9$, and $20.0 \mathrm{~nm}$ are simulated. For each width and twin spacing combination, five sample thicknesses, from 8, 24, 50, 100, 200 layers of atoms along [110] direction are simulated, which correspond to a NW thickness of $1.02 \mathrm{~nm}$ (plane strain), $3.1 \mathrm{~nm}, 6.4 \mathrm{~nm}, 12.8 \mathrm{~nm}$, and $25.6 \mathrm{~nm}$, respectively.

The embedded atom method potential ${ }^{25}$ for $\mathrm{Cu}$ is applied. All simulations are performed using NVE ensemble (constant number of atoms $\mathrm{N}$, volume $\mathrm{V}$, and total energy $\mathrm{E}$ ) in lammps ${ }^{26}$ and started at $300 \mathrm{~K}$. A constant time step of 2 femto-seconds is used. Uniaxial tension is applied along $z$ axis. In plane strain deformation, periodic boundary condition is applied along the thickness direction ( $y$ axis in Fig. 1(a)), and a sample thickness of $h=1.02 \mathrm{~nm}$ is used. For three-dimensional simulations with different sample thicknesses, no constraints are applied to all sides except the top and bottom ends. A periodic boundary is used for those two ends during straining. Before deformation, each sample is relaxed for 100 picoseconds using an NPT ensemble to ensure pressure approaches to nearly zero during this period. Also, to apply NPT ensemble in three-dimensional wires with free surfaces, a vacuum space of $3 \mathrm{~nm}$ is left at the 


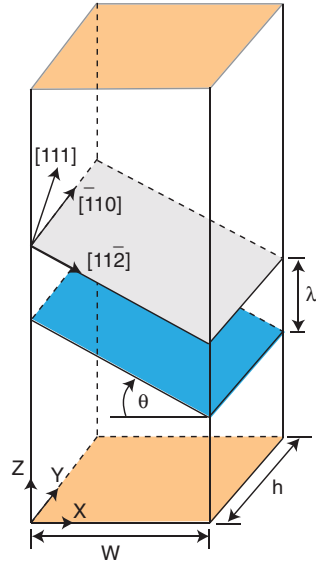

(a)

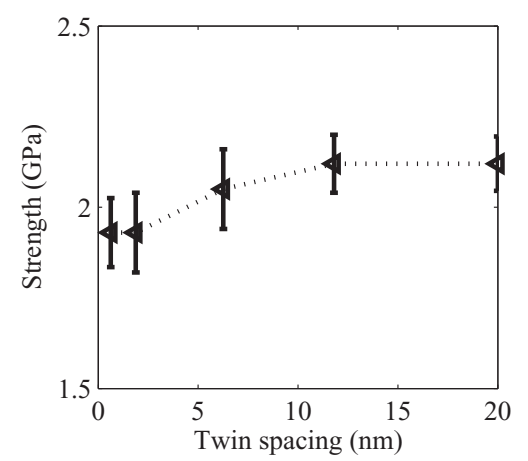

(b)
FIG. 1. (Color online) Nanowires with tilted twins in tension. (a) The geometry of a face center cubic nanowire with thickness

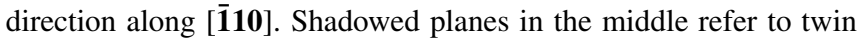
boundaries, and twin planes are tilted by an angle $\theta$ with respect to the wire/loading axis. Twins are spaced by a uniform distance $\lambda$. (b) Average flow stresses versus twin spacings for NWs of $W=30$ $\mathrm{nm}$ and $h=25.6 \mathrm{~nm}$. For here and in what follows, flow stresses are averaged over engineering strain from $5 \%$ to $20 \%$ from a stress-strain curve; error bars represent the standard deviation from statistical analyses with 150 sampling points.

side of a free surface except the two ends. A $20 \%$ strain is applied to each relaxed sample in 400 picoseconds, which corresponds to an applied strain rate of $5 \times 10^{8} / \mathrm{s}$.

\section{RESULTS FROM MOLECULAR DYNAMICS SIMULATIONS}

Figure 1(b) shows the average flow stresses in NWs of width $W=30 \mathrm{~nm}$, thickness $h=25.6 \mathrm{~nm}$, and twin tilt angle $\theta=30^{\circ}$ for different twin spacings. It indicates that when sample width and thickness are fixed, the average flow stresses decrease as twin spacings are reduced. This behavior is reminiscent of strength softening in nanotwinned $\mathrm{Cu}$ as the twin spacing is reduced to below a critical value. ${ }^{27-30}$ To investigate the dependence of average flow stresses on sample sizes, we fix the sample width $W=30 \mathrm{~nm}$ and twin spacing $\lambda$ while changing the thickness $h$. The stress-strain curves for samples with twin spacing $\lambda=20.0 \mathrm{~nm}$ are shown in Fig. 2(a). There is no apparent change for average flow stresses as the wire thicknesses are reduced (Fig. 2(b)).

Note that the size-insensitive behavior in NWs with tilted twins shown in Fig. 2 is for samples with different thicknesses $h$ but fixed $W$ and $\lambda$. To investigate the size effect due to proportional reduction in sample width $W$, thickness $h$, and twin spacing $\lambda$, we simulate samples with different widths $W$ at fixed width-to-thickness $(W / h)$ and width-to-twin-spacing ratios $(W / \lambda)$. Stress-strain curves for wire width $W=30 \mathrm{~nm}$, $21 \mathrm{~nm}, 12 \mathrm{~nm}$, and $6 \mathrm{~nm}$ are shown in Fig. 3. Curves in Fig. 3(a) are for samples with constant ratios of $W / h=1.2$ and $W / \lambda=1.5$ (left) and those with $W / h=5$ and $W / \lambda=5$ (right). The samples are truly self-similar in that the length-to-width ratio is also kept constant. Compared to the case of reducing $h$ alone, the self-similar samples exhibit strengthening in that
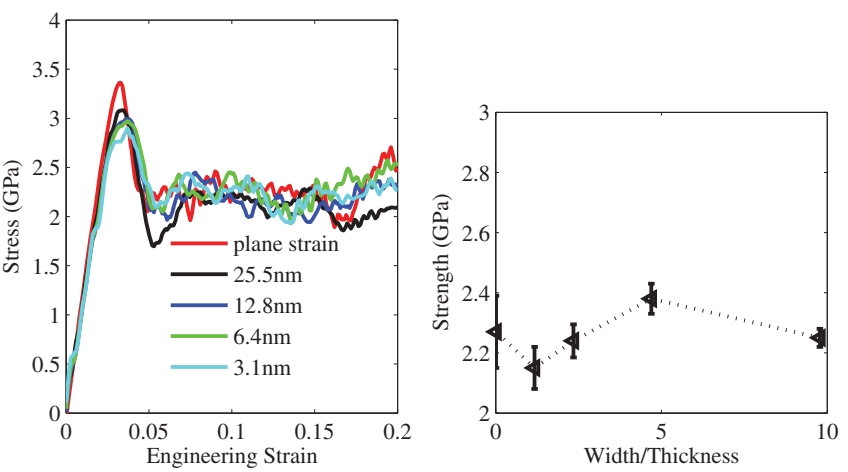

FIG. 2. (Color online) Stress-strain behaviors for NWs with $W=30 \mathrm{~nm}$ and $\lambda=20 \mathrm{~nm}$ as their thicknesses decrease. (a) Stress-strain curves. (b) Average flow stresses versus sample width. No apparent size effect in strength is observed if we only reduce sample thicknesses.

average flow stresses increase as the sample dimensions are proportionally reduced (Fig. 3(b)).

Typical stress-strain curves of NWs with tilted twins in Fig. 3(a) show that the flow stress remains almost constant from about $5 \%$ to $20 \%$ strains after a load drop immediately following the peak strength. This load drop can be attributed to the very high strain rates in MD simulations, which could be reduced or eliminated by decreasing the loading rate. ${ }^{31}$ The nearly constant flow stress in the stress-strain curves after 5\% strain is associated with migration of twin boundaries in the NWs, as seen in Fig. 4. The maximum axial plastic strain by twin migration in those NWs could reach $\varepsilon_{t w}^{p}=f e^{\mathbf{T}} \sin \theta$ when the NWs are subjected to tension, where $f$ is the volume fraction of initially twinned region, $\theta$ the tilt orientation of twin planes, and $e^{\mathrm{T}}$ the characteristic shear strain of detwinning. For face-centered cubic metals with (111) [112] twins, $e^{\mathrm{T}}=0.707$, and the maximum plastic strain by detwinning in the sample (in Fig. 1(a)) is about $\varepsilon_{t w}^{p}=0.18$ for $f=0.5$ and $\theta=30^{\circ}$. One could thus realize different amount of ductility in NWs with tilted twins by manipulating the volume fraction of twins and tilt angles. After the depletion of twins, a deformed NW

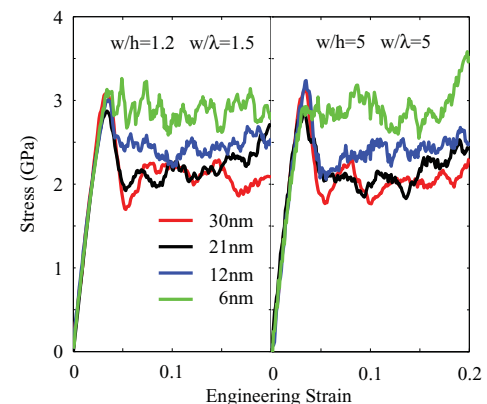

(a)

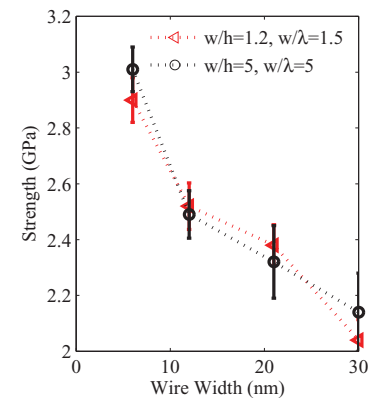

(b)
FIG. 3. (Color online) Strengthening in self-similar wires with sample width from 30 to $6 \mathrm{~nm}$ while keeping $W / h$ and $W / \lambda$ ratios constant. (a) Stress-strain curves for several wire widths: $W / h=1.2$ and $W / \lambda=1.5$ (left) and $W / h=5$ and $W / \lambda=5$ (right). (b) Average flow stresses versus wire width for $W / h=1.2$ and $W / \lambda=1.5$ and $W / h=5$ and $W / \lambda=5$. Smaller-being-strong size effect in strength is observed if we reduce all sample dimensions proportionally. 


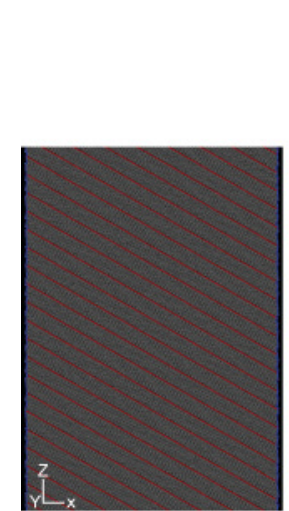

(a)

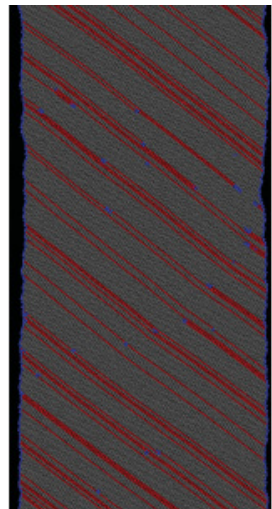

(b)

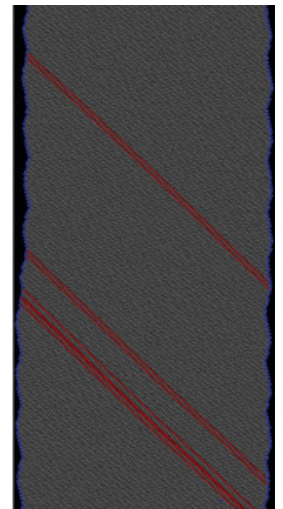

(c)
FIG. 4. (Color online) Detwinning in $W=30 \mathrm{~nm}$ and $\lambda=1.88 \mathrm{~nm}$ NWs (plane strain deformation). Here color grey, red, and blue are used to identify perfect atoms, atoms in stacking faults, and atoms of other types, respectively. (a) The sample after relaxation; (b) atomic snapshot of the sample deformed by $10 \%$ strain; (c) atomic snapshot of the sample deformed by $20 \%$ strain.

becomes a single crystal and is almost defect free, as seen in Fig. 4.

Note that by decreasing the twin spacing $\lambda$ alone, the NWs show relatively minor softening (Fig. 1(b)) due to increasing nucleation sources; while for proportional reduction in all sample dimensions of NWs (see Fig. 3(a)), the drop in strength is substantial. This difference cannot be attributed to changes in the density of dislocation nucleation sites. We check the detailed dislocation patterns in order to find the distinct strengthening mechanisms. Dislocation patterns at $\sim 10 \%$ strain in samples with $W=30 \mathrm{~nm}$ and $\lambda=$ $1.88 \mathrm{~nm}$ are shown in Fig. 5(a) for sample thicknesses of $h=25.6 \mathrm{~nm}$. Dislocations in the sample are nucleated from the ledges of NWs, and most of them are highly curved with one end moving along the [112] direction and the other

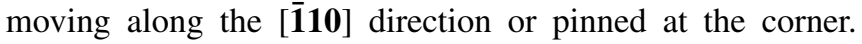
It is also found that both ends of dislocations could be pinned at the free surfaces while their loops extend (the dislocation marked as D-loop in Fig. 5(a)). Figures 5(b) to 5(e) present the entire process of nucleation, bow-out, gliding, and absorption of one dislocation. Nucleation of the partial dislocation commonly starts at the junction of a twin plane and a wire ledge (Fig. 5(b)). Under uniaxial tension, the dislocation extends in the twin boundary (Fig. 5(c)), with one end moving along [112] direction ( $x$ axis) and the other gliding along [110]direction ( $y$ axis). As one end of the dislocation is close to the side wall, it tends to be pinned by the side wall (Fig. 5(d)). Due to probable surface effect, the two ends of the dislocation move slower, and the dislocation bows out. At the end, the whole dislocation is absorbed by the opposite side of the NW (Fig. 5(e)). The formation of a new partial dislocation at the surface-twin boundary junction is also seen in Fig. 5(e).

The bow-out of twinning partials in the samples show evidence of twin boundaries temporarily pinned at free surfaces during partial-dislocation-induced migration. Therefore, we speculate that depinning of partial dislocations in free surfaces plays a central role to account for the size effect

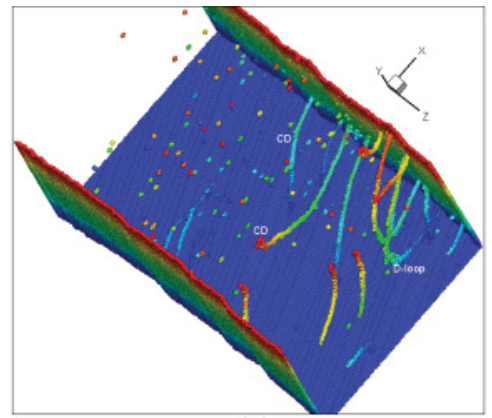

(a)

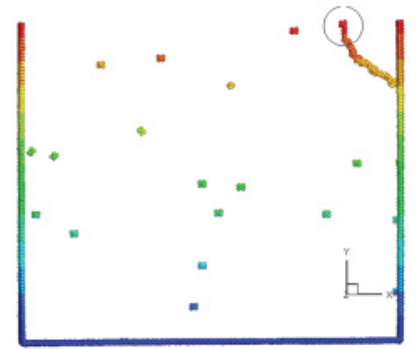

(b)

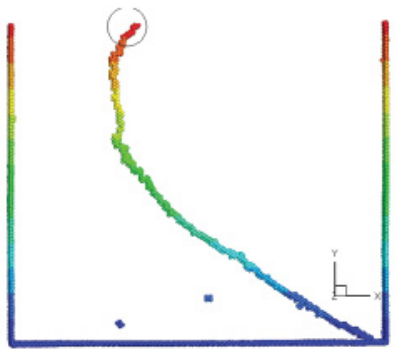

(d)

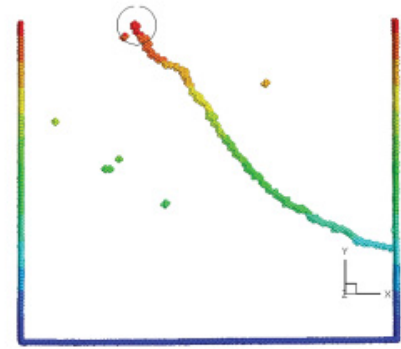

(c)

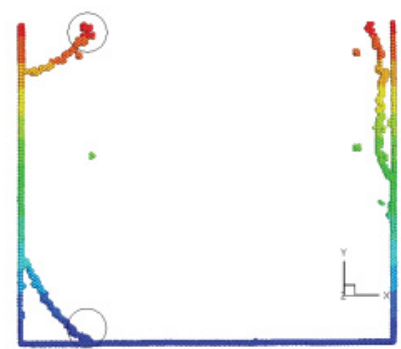

(e)
FIG. 5. (Color online) Dislocation activities in $W=30 \mathrm{~nm}$, $h=25.6 \mathrm{~nm}$, and $\lambda=1.88 \mathrm{~nm}$ NWs at $10 \%$ strain. (a) Dislocation pattern (CD: curved dislocation, D-loop: dislocation loop). (b) to (e) Nucleation, extension, bow-out, and absorption of a twinning partial in the twin plane. (b) Formation of the twinning partial from the sample edge. (c) Extension of the twinning partial, with one end

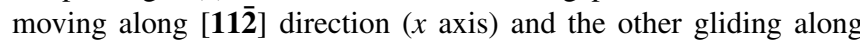
[110] direction ( $y$ axis). (d) One end being pinned by the side wall as the dislocation bows out. (e) Absorption of the twinning partial at the opposite surface.

for strength. As the ends of a twinning partial dislocation are pinned by free surfaces, dislocation propagation requires dislocation depinning along the two (110) side walls, and how the side walls influence depinning become crucial. Hence the width of the sample plays an important role for strengthening. The sample thickness (along [110] direction) is primarily associated with dislocation nucleation. If a twinning partial along [110] pinned at two ends in the slip plane bulges between them during its nucleation, as seen in the Frank-Read type sources, we expect that the strength of NWs with tilted twins would show a strength dependence of $1 / h$. The following finite element simulations, combined with dislocation nucleation patterns seen in Fig. 5, would alternatively suggest that dislocations are nucleated from NW ledges due to stress concentration, which results in different strength scaling to that described by Frank-Read sources. 


\section{FINITE ELEMENT SIMULATIONS}

In this section, we employ single-crystal plasticity model to understand the stress and strain distribution in twinned NWs. A similar sample shown in Fig. 1(a) is adopted with tilted angle of twin planes about $37^{\circ}$. Note here $y$ axis is along [ $\overline{\mathbf{1 1 0}}$ ] direction, and $z$ axis is the loading axis. In this laboratory coordinate, the Euler angles $(\varphi, \theta, \omega)$ of the matrix material and the twinned regime are $\left(-45^{\circ},-91.6^{\circ}, 90^{\circ}\right)$ and $\left(-45^{\circ},-17.9^{\circ},-90^{\circ}\right)$, respectively. We employ the classical framework of rate-dependent single-crystal plasticity ${ }^{32,33}$ to model plasticity by gliding of leading partial dislocations. In such framework, the deformation gradient $\boldsymbol{F}$ is decomposed into elastic $\left(\boldsymbol{F}^{\mathrm{e}}\right)$ and plastic $\left(\boldsymbol{F}^{\mathrm{p}}\right)$ parts as

$$
\boldsymbol{F}=\boldsymbol{F}^{\mathrm{e}} \boldsymbol{F}^{\mathrm{p}}
$$

with $\operatorname{det}\left(\boldsymbol{F}^{\mathrm{e}}\right)>0$ and $\operatorname{det}\left(\boldsymbol{F}^{\mathrm{p}}\right)=1$. Plastic flow takes place through slip of leading partial dislocations on prescribed crystallographic slip systems, with each system $\alpha$ defined by a slip direction $\boldsymbol{S}^{\alpha}$ and a slip-plane normal $\boldsymbol{m}^{\alpha}$. The Green elastic strain measure $\boldsymbol{E}^{e}$ and the symmetric second Piola-Kirchhoff stress tensor $\boldsymbol{T}$ are hence defined respectively as

$$
\boldsymbol{E}^{\mathrm{e}}=(1 / 2)\left(\boldsymbol{F}^{\mathrm{eT}} \boldsymbol{F}^{\mathrm{e}}-1\right) \text { and } T \equiv \boldsymbol{C} E^{\mathrm{e}}
$$

where the superscript $\mathrm{T}$ stands for the transpose of a tensor and $\boldsymbol{C}$ is the fourth-order tensor of elastic moduli. The evolution of the plastic deformation gradient, the flow rule, is described by

$$
\dot{\boldsymbol{F}}^{p} \boldsymbol{F}^{p-1}=\sum_{\alpha} \dot{\gamma}^{\alpha} \frac{\boldsymbol{S}^{\alpha} \otimes \boldsymbol{m}^{\alpha}+\boldsymbol{m}^{\alpha} \otimes \boldsymbol{S}^{\alpha}}{2}
$$

where $\dot{\gamma}^{\alpha}$ is the shearing rate on the $\alpha$ th slip system. A phenomenological description of plastic flow in an NW with tilted twins is used, which is the rate-dependent law suggested by Asaro and Needleman ${ }^{32}$ as

$$
\dot{\gamma}^{\alpha}=\left\{\begin{array}{cl}
\dot{\gamma}_{0}\left(\frac{\tau^{\alpha}}{\tau_{c}}\right)^{1 / n} & \text { when } \tau^{\alpha}>0 \\
0 & \text { if } \tau^{\alpha}<0
\end{array}\right.
$$

where $\dot{\gamma}_{0}$ and $n$ denote a referential strain rate and strain-rate sensitivity associated with dislocation motion; $\tau_{c}$ is the slip resistance to leading partial dislocations and $\tau^{\alpha}$ is the resolved shear stress on the $\alpha$ th slip system given as

$$
\tau^{\alpha}=\left(\boldsymbol{F}^{\mathrm{eT}} \boldsymbol{F}^{\mathrm{e}} \boldsymbol{T}\right)\left(\boldsymbol{S}^{\alpha} \otimes \boldsymbol{m}^{\alpha}\right)
$$

For simplicity, it is assumed that there is no hardening for slip resistances. Note that in the presence of twins, a partial dislocation is trailed by detwinning of one layer of atoms in a twin plane rather than generating a stacking fault. For the latter, the excessive stacking fault energy requires a threshold stress of $\gamma_{s f} / b_{\mathrm{p}}$ to initiate plastic flow by partial dislocations, where $\gamma_{s f}$ is the stacking fault energy and $b_{\mathrm{p}}$ the Burgers vector of a partial dislocation. Corresponding crystal plasticity subroutine implemented by Kalidindi et al. ${ }^{33}$ in finite element software Abaqus ${ }^{34}$ is used for our simulation. Plastic slips exclusively come from the three (111) [112] twin systems in the twin plane with slip resistance $\tau_{\text {crit }}$. For partial dislocations inclined to twin planes, their nucleation and propagation will be inevitably trailed by a stacking fault and twin boundaries could serve as barriers to their motion. Hence we use much higher slip resistances for partial dislocations inclined to twin

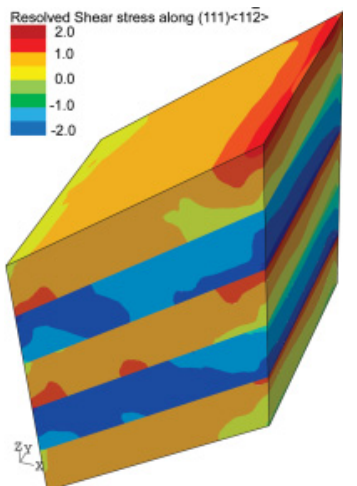

(a)

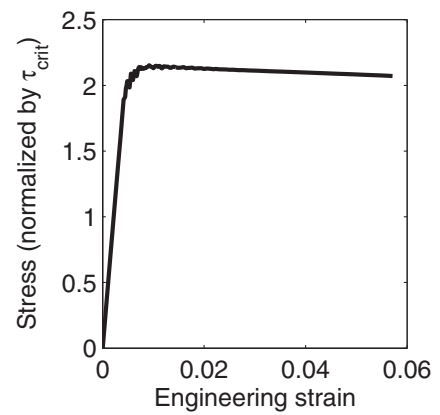

(b)

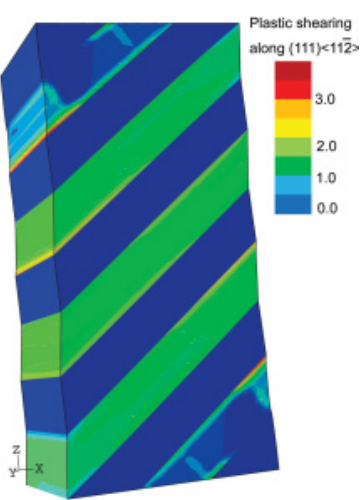

(c)
FIG. 6. (Color online) Finite-element simulations on the deformation in wires with tilted twins. (a) Contour for resolved shear stress in (111) plane along [112] direction before plastic deformation. Only the middle portion of the sample is shown. The stress is normalized by the macroscopic average shear stress in (111) [112̄] slip system. There is clear stress-concentration at the junctions of a twin plane and sample surfaces. (b) Stress-strain curve from finite element simulation. After the initial yielding, a constant flow stress is shown. (c) Contour for resolved plastic shearing strain in (111) plane along [112] direction at the end (normalized by the applied 5\% total strain). It is noted that due to the polar nature of plastic shearing by leading partial dislocations in (111) [112] systems, plastic deformation only occurs in those twinned regimes.

planes than resistance to twinning partials. A uni-axial tension at a strain rate of $10^{-4} / \mathrm{s}$ is applied to the sample along $z$ axis. The sample contains a total of 218160 three dimensional elements with reduced integration points. Material parameters used to model the mechanical behavior in $\mathrm{Cu}$ NWs with tilted twins are given here. Elastic constants for single-crystal $\mathrm{Cu}$ are: $C_{11}=170 \mathrm{GPa}, C_{12}=120 \mathrm{GPa}$, and $C_{44}=75 \mathrm{GPa} ;{ }^{35}$ material parameters for plastic slip rates are: $\dot{\gamma}_{0}=10^{-7} / \mathrm{s}$, $n=0.005$. Since we are interested in the competition of plastic deformation in the two types of dislocation motion, inclined ones versus those parallel to twin planes, the choices for the two parameters for plastic slip rates are not crucial.

Figure 6(a) presents the resolved shear stress contour for the (111) [112] slip system at $0.3 \%$ strain, where the whole sample is still in the elastic region. We show the middle portion of the sample for clarity. Stress concentration at the junctions of twin planes and sample surfaces is clearly seen. After the initial yielding, a constant flow stress is seen in the stress-strain curve (Fig. 6(b)). Corresponding contour for resolved plastic 
shearing strain in the slip system of (111) [112] (normalized by the applied 5\% total strain) is given in Fig. 6(c). Due to the polar nature of plastic shearing by leading partial dislocations

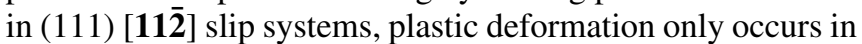
those twinned regimes. The finite element simulation confirms that there is strong stress concentration at the junction of twin boundaries and surface ledges, which is consistent with other MD simulations. ${ }^{36}$ Stress concentration-driven nucleation at sample ledges indicates that dislocation nucleation sites will be constant as the sample thickness varies or all dimensions are proportionally reduced, and this mechanism would not give rise to apparent size effect. In the next section, we attempt to explain why the width of NWs in Fig. 1(a) (the dimension along dislocation propagation) could significantly influence the strength of such NWs by deriving the surface attraction to nucleated dislocations.

\section{IMAGE FORCE ON NUCLEATED DISLOCATIONS IN NANOWIRES}

Since the thickness has minor effect to strength for NWs shown in Fig. 1, we further explore the surface effect at dislocation propagation stage as the width of the NWs changes. Surface stress alone is not regarded as the primary course since it only influences NWs with very small diameters. ${ }^{37}$ We are interested in the interactions between dislocations and free surfaces. Exact mathematical expressions of the energy variance associated with dislocation motion in NWs with tilted twins is difficult: (a) the formula of dislocation induced displacement-stress fields in anisotropic elastic media is already very tedious; (b) the presence of twin boundaries and free surfaces in those wires complicate the analysis; and (c) the applicability of continuum dislocation theory for wires with typical dimensions of tens of nanometers is still under debate. For practical reasons, we consider the special case of an isotropic thin film with $h \rightarrow \infty$ to obtain the surface attraction to a dislocation inside the film. For the edge dislocation (in blue) in the film shown in Fig. 7, it is convenient to construct two dislocation arrays such that all stresses acting on the two

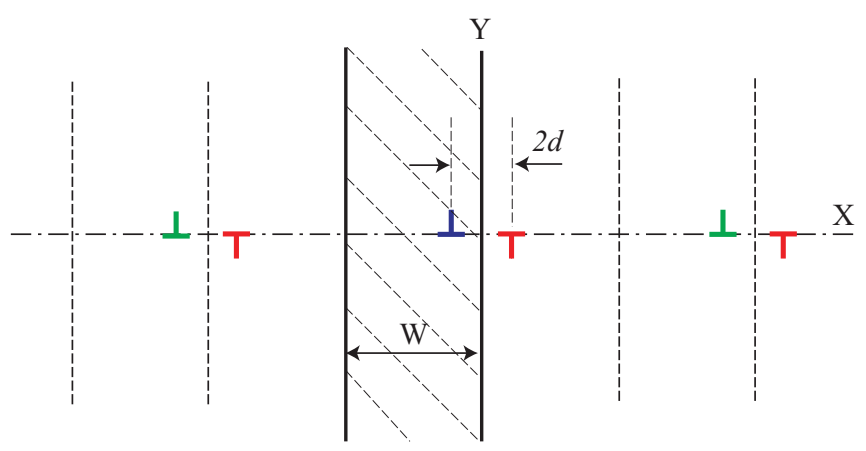

FIG. 7. (Color online) Construction of infinite arrays of image dislocations (in red and green) to ensure traction-free surfaces in the thin film with width $W$ when a dislocation is nucleated (in blue). Those image dislocations in red would result in a net force $F_{x}$ to the nucleated dislocation; and the green ones would cancel each other and have no contribution to $F_{x}$. surfaces of the thin films vanish except $\sigma_{x y}$. At the right side free surface, we have

$$
\begin{aligned}
\sigma_{x y}(0, y)= & \frac{\mu b}{\pi(1-v)}\left\{\frac{d\left(d^{2}-y^{2}\right)}{\left(d^{2}+y^{2}\right)^{2}}\right. \\
& \left.-\sum_{n=1}^{\infty}\left[\frac{L_{n}\left(L_{n}^{2}-y^{2}\right)}{\left(L_{n}^{2}+y^{2}\right)^{2}}-\frac{l_{n}\left(l_{n}^{2}-y^{2}\right)}{\left(l_{n}^{2}+y^{2}\right)^{2}}\right]\right\}
\end{aligned}
$$

where $L_{n}=2 n W-d$ and $l_{n}=2 n W+d, \mu$ and $v$ are the shear modulus and the Poisson's ratio of the film, and $b$ is the Burgers vector of the dislocation. For the left-side surface, the shear stress could be given as

$$
\begin{aligned}
& \sigma_{x y}(-W, y) \\
& =\frac{\mu b}{\pi(1-\nu)} \sum_{n=1}^{\infty}\left\{\frac{R_{n}\left(R_{n}^{2}-y^{2}\right)}{\left(R_{n}^{2}+y^{2}\right)^{2}}-\frac{r_{n}\left(r_{n}^{2}-y^{2}\right)}{\left(r_{n}^{2}+y^{2}\right)^{2}}\right\}
\end{aligned}
$$

where $R_{n}=(2 n-1) W+d$ and $r_{n}=(2 n-1) W-d$. Both $\sigma_{x y}(0, y)$ and $\sigma_{x y}(-W, y)$ converge to finite values quickly with increasing $n$. These fictitious stresses in Eq. (6) could be cancelled by adding a serial of stress functions, ${ }^{38}$ which is beyond the scope of this work because each individual stress function has no contribution to the net pulling force $F_{x} .{ }^{38} \mathrm{We}$ see that those image dislocations in red would result in a net force $F_{x}$ to the edge dislocation in the film; and the green ones would cancel each other and have not contribution to $F_{x}$. The force per unit length exerted to the edge dislocation in Fig. 7 from all image dislocations can be expressed as

$$
F_{x}=\sum_{n=-\infty}^{\infty} \frac{\mu b^{2}}{2 \pi(1-v)} \frac{1}{2 n W+2 d}=\frac{\mu b^{2}}{4(1-v)} \frac{1}{W} \cot \left(\frac{\pi d}{W}\right)
$$

Considering that during the nucleation of dislocations in a NW, a dislocation usually forms a curved loop with two ends pinned at the free surfaces (see Fig. 5), its distance to the free surface is about a fraction of the NW's width, i.e. $d \propto W$. Equation (6b) could be simplified to follow $F_{x} \propto 1 / W$. The critical shearing strength to drive the dislocation moving forward is hence proportional to $\mu b / W$, which is consistent with our MD simulations that the strength of NWs becomes stronger as the sample size is reduced proportionally.

\section{CONCLUDING REMARKS}

To conclude, we presented one-dimensional coherent nanostructures which could help to develop straightforward understanding on the origin of size effect in strength. We reveal that strengths in $\mathrm{Cu} N W s$ with tilted twins show no apparent change if only their thicknesses along [ $\mathbf{1} 10]$ direction reduce, i.e. the dimension along dislocation line is not critical for strength in this particular wire structure; NWs become stronger as their sample sizes are reduced proportionally, i.e. the dimension along the dislocation propagation direction is crucial for strength. Nucleation site density is not regarded as the major course of such size effects. Stress concentrations at the edge of NWs with rectangular cross sections are speculated to be the course of the insensitive strength dependence on 
NW thickness along [110] direction. Probably stronger surface pinning of partial dislocation in samples with smaller width could be the main reasons behind the size effects in those NWs. Our analysis on surface attraction to nucleated dislocations in the NW shows that surface attraction becomes stronger as wire width reduces. In addition, we show that NWs with tilted twins exhibit elastic, almost perfectly plastic behavior upon straining, and a large amount of plasticity could be achieved at room temperature by detwinning. This study suggests an alternative approach to modulating strength and stretchability/ductility in one-dimensional coherent nanostructures, in a way similar to those broadly explored for stretchable electronics. ${ }^{39}$ Current success in manipulating microstructures during NW growth ${ }^{40-42}$ might make direct growth of such NWs with tilted twins feasible.

\section{ACKNOWLEDGMENTS}

The author is very grateful to Huajian Gao and Allan F. Bower of Brown University for fruitful discussions. This work was supported in part by the Hundred Talent Program and by NSFC No. 11021262. Computation was supported by the Super Computing Center of CAS and the Institute of Mechanics. *yujie_wei@lnm.imech.ac.cn

${ }^{1}$ M. D. Uchic, D. M. Dimiduk, J. N. Florando, and W. D. Nix, Science 305, 986 (2004).

${ }^{2}$ J. R. Greer, W. C. Oliver, and W. D. Nix, Acta Mater. 53, 1821 (2005).

${ }^{3}$ B. Wu, A. Heidelberg, and J. J. Boland, Nat. Mater. 4, 525 (2005). ${ }^{4}$ E. Ma, T. D. Shen, and X. L. Wu, Nat. Mater. 5, 515 (2006).

${ }^{5}$ Z. W. Shan, R. K. Mishra, S. A. Syed Asif, O. L. Warren, and A. M. Minor, Nat. Mater. 7, 115 (2008).

${ }^{6}$ S. Brinckmann, J. Y. Kim, and J. R. Greer, Phys. Rev. Lett. 100, 155502 (2008).

${ }^{7}$ Q. Yu, Z. W. Shan, J. Li, X. Huang, L. Xiao, J. Sun, and E. Ma, Nature 463, 335 (2010).

${ }^{8}$ A. T. Jennings, M. J. Burek, and J. R. Greer, Phys. Rev. Lett. 104, 135503 (2010).

${ }^{9}$ E. A. Withey, A. M. Minor, D. C. Chrzan, J. W. Morris Jr., and S. Kuramoto, Acta Mater. 58, 2652 (2010).

${ }^{10}$ C. M. Byer, B. Li, B. Cao, and K. T. Ramesh, Scr. Mater. 62, 536 (2010).

${ }^{11}$ E. Lilleodden, Scr. Mater. 62, 532 (2010).

${ }^{12}$ G. Dehm, Prog. Mater. Sci. 54, 664 (2009).

${ }^{13}$ T. Zhu and J. Li, Prog. Mater. Sci. 55, 710 (2010).

${ }^{14}$ H. S. Park, K. Gall, and J. A. Zimmerman, Phys. Rev. Lett. 95, 255504 (2005).

${ }^{15}$ W. W. Liang, M. Zhou, and F. J. Ke, Nano. Lett. 5, 2039 (2005).

${ }^{16}$ W. Liang and M. Zhou, Phys. Rev. B 73, 115409 (2006).

${ }^{17}$ H. S. Park, Nano Lett. 6, 958 (2006).

${ }^{18}$ L. Sandoval and H. M. Urbassek, Nano Lett. 9, 2290 (2009).

${ }^{19}$ A. J. Cao and Y. G. Wei, J. Appl. Phys. 102, 083511 (2007).

${ }^{20}$ C. Deng and F. Sansoz, Nano Lett. 9, 1517 (2009).

${ }^{21}$ L. Li and N. M. Ghoniem, Phys. Rev. B 79, 075444 (2009).
${ }^{22}$ J. R. Greer, D. Jang, J. Y. Kim, and M. J. Burek, Adv. Func. Mater. 19, 2880 (2009).

${ }^{23}$ J. Rajagopalan, J. Han, and M. T. A. Saif, Science 315, 1831 (2007).

${ }^{24}$ D. Kiener, W. Grosinger, G. Dehm, and R. Pippan, Acta Mater. 56, 580 (2008).

${ }^{25}$ Y. Mishin, M. J. Mehl, D. A. Papaconstantopoulos, A. F. Voter, and J. D. Kress, Phys. Rev. B 63, 224106 (2001).

${ }^{26}$ S. J. Plimpton, J. Comp. Phys. 117, 1 (1995).

${ }^{27}$ L. Lu, X. Chen, X. Huang, and K. Lu, Science 323, 607 (2009).

${ }^{28}$ X. Li, Y. Wei, K. Lu, L. Lu, and H. Gao, Nature 464, 877 (2010).

${ }^{29}$ Y. Wei, Mater. Sci. Eng. A 528, 1558 (2011).

${ }^{30}$ Y. Wei, Phys. Rev. B 83, 132104 (2011).

${ }^{31}$ J. Schiøtz and K. W. Jacobsen, Science 301, 1357 (2003).

${ }^{32}$ R. J. Asaro and A. Needleman, Acta Metall. 33, 923 (1985).

${ }^{33}$ S. R. Kalidindi, C. A. Bronkhorst, and L. Anand, J. Mech. Phys. Solids 40, 537 (1992).

${ }^{34}$ Abaqus, Abaqus Users Manual. Simulia Co. (2008).

${ }^{35} \mathrm{G}$. Simmons and H. Wang, Single Crystal Elastic Constants and Calculated Aggregate Properties: A Handbook, P22 (The MIT Press, Cambridge, MA, 1971).

${ }^{36}$ T. Zhu, J. Li, A. Samanta, A. Leach, and K. Gall, Phys. Rev. Lett. 100, 025502 (2008).

${ }^{37}$ J. Diao, K. Gall, and M. L. Dunn, Nano Lett. 4, 1863 (2004).

${ }^{38}$ J. P. Hirth and J. Lothe, Theory of Dislocations, 2nd ed. (John Wiley \& Sons, Inc, 1982) p. 86, Chap. 3.

${ }^{39}$ J. A. Rogers, T. Someya, and Y. Huang, Science 327, 1603 (2010).

${ }^{40}$ C. Wang, Y. Wei, H. Jiang, and S. Sun, Nano Lett. 10, 2121 (2010).

${ }^{41}$ Q. Xiong, J. Wang, and P. C. Eklund, Nano Lett. 6, 2736 (2006).

${ }^{42}$ J. Johansson, L. S. Karlsson, C. P. T. Svensson, T. Martensson, B. A. Wacaser, K. Deppert, L. Samuelson, and W. Seifert, Nat. Mater. 5, 574 (2006). 